\title{
Prévalence des principales bactéries responsables de mammites subcliniques des vaches laitières au nord-est de I'Algérie
}

\author{
Z. Boufaida Asnoune ${ }^{1 *}$ M.J. Butel ${ }^{2}$ R. Ouzrout ${ }^{1}$
}

\section{Mots-clés}

Bovin - Vache laitière - Mammite Infection latente - Analyse bactériologique - Algérie.

\begin{abstract}
Résumé
Des analyses bactériologiques de 235 prélèvements de lait de vaches laitières atteintes de mammites subcliniques dépistées par le California mastitis test, provenant de dix exploitations du nord-est de l'Algérie, ont été réalisées sur une période de deux ans (2008-10). L’isolement bactérien a concerné 89 p. 100 des échantillons. Parmi les germes isolés, les coques à Gram positif ont été les plus fréquents, notamment des staphylocoques à coagulase négative (43 p. 100) et Staphylococcus aureus (30 p. 100). Escherichia coli a été isolé dans 14 p. 100 des prélèvements. Les autres germes isolés ont été Streptococcus uberis (4 p. 100), Streptococcus agalactiae (3 p. 100), Streptococcus dysgalactiae (3 p. 100), et Klebsiella sp. plus rarement (2 p. 100).
\end{abstract}

La mammite est considérée comme l'une des pathologies les plus importantes, fréquentes et coûteuses affectant les vaches laitières $(10,11)$, et la plus pénalisante pour les élevages laitiers (22). Elle se traduit le plus souvent sous forme inapparente, sans symptôme ni altération visible du lait. Biologiquement, elle se manifeste seulement par une augmentation du taux des leucocytes et des cellules épithéliales détectées par divers tests de comptage cellulaire. Ces tests permettent d'apprécier l'état sanitaire de la mamelle et d'évaluer la qualité du lait destiné à la consommation.

Dans de nombreux pays développés, une mise sous surveillance systématique et régulière est entreprise au sein des élevages laitiers afin de dépister les cas de mammites. En revanche, en Algérie la plupart des élevages ne sont soumis à aucun contrôle laitier régulier. La fréquence des mammites cliniques et subcliniques est élevée (6). Selon le ministère de l'Agriculture et du Développement rural, l'Algérie a un cheptel de 900000 vaches laitières, dont 230000 bovins laitiers à haut potentiel.

1. Institut des sciences vétérinaires, centre universitaire El Tarf, BP 73, 36000 Algérie.

2. Faculté des Sciences pharmaceutiques et biologiques, laboratoire de microbiologie, Université Paris 5, France.

* Auteur pour la correspondance

Tél. : +213659482929

E-mail : zahidaboufaida@hotmail.fr
L'Algérie, troisième importateur mondial après l'Italie et le Mexique, a une consommation moyenne de lait de $130 \mathrm{~L} / \mathrm{habi}$ tant/an (3), dont le montant s'élève à 600 millions de dollars pour importer la moitié de ses besoins, soit 5 milliards de litres. En 2009 , ce pays a importé de la poudre de lait pour un montant de 350 millions de dollars. Afin de réduire les coûts d'importation, les pouvoirs publics ont opté pour l'importation massive de vaches laitières, mais l'augmentation de la production laitière doit aussi s'accompagner d'une amélioration de l'état sanitaire des vaches. La présente étude a eu pour objectif d'évaluer la prévalence de mammites subcliniques dans des exploitations laitières, afin d'identifier les germes responsables de ces infections.

\section{MATERIEL ET METHODES}

\section{Exploitations}

L'étude a été conduite sur une période de deux années dans dix exploitations situées dans cinq wilayas du nord-est de l'Algérie (EL Tarf, Annaba, Skikda, Guelma et Souk haras). Les critères de choix des exploitations ont été la facilité d'accès et la disponibilité des éleveurs. Une enquête épidémiologique a été entreprise par le biais d'une fiche de renseignements relatifs à la conduite de la traite, à son hygiène et au traitement au tarissement.

\section{Prélèvements}

$\mathrm{Au}$ total 248 vaches ont été incluses dans cette étude. Les prélèvements de lait ont été effectués directement à la mamelle, avant 
la traite du soir. Deux prélèvements ont été réalisés sur chaque quartier : le premier a servi à dépister les mammites subcliniques au pied de la vache avec le California mastitis test (CMT). Le deuxième a été destiné à l'analyse bactériologique et n'a concerné que les échantillons de lait détectés positifs par le CMT.

Pour le deuxième prélèvement, le lait a été collecté dans un flacon stérile après lavage à l'eau et désinfection des trayons avec de l'alcool à $70^{\circ}$ et élimination des premiers jets, selon les recommandations du National Mastitis Council (19). Tous ces prélèvements ont été identifiés et acheminés au laboratoire dans des conditions strictes de réfrigération $\left({ }^{\circ} \mathrm{C}\right)$ dans un délai de 18 heures maximum (4).

\section{Dépistage des mammites par le California mastitis test}

Le principe du CMT repose sur l'utilisation d'une solution à base d'alkylaryl sulfonate de sodium à 4 p. 100 avec du pourpre de bromocrésol à 1/10 000 qui joue le rôle d'indicateur de $\mathrm{pH}$ (5). Trois millilitres de lait à tester sont mélangés à une même quantité de réactif (Schalm-Test, Rhône Mérieux).

Le score du CMT va de 0 à 4 en fonction de l'aspect du mélange (14). La relation entre le nombre de cellules et le score du CMT a été établie (tableau I) pour permettre le dépistage de l'infection du quartier. Ce test est considéré positif à partir d'un score de 2 . Si au moins un quartier est positif, la vache est déclarée positive et si tous les quartiers sont négatifs, la vache est déclarée saine.

\section{Analyses bactériologiques du lait}

L'ensemencement des échantillons positifs au CMT a été réalisé sur gélose au sang de mouton (5 p. 100) et sur gélose Hektoen incubés à $37^{\circ} \mathrm{C}$ pendant $24-48$ heures. L'identification des bactéries a été effectuée par les méthodes conventionnelles (aspect des colonies, coloration de Gram, test à la catalase, test à l'oxydase associé à la coagulase). Les caractères biochimiques ont été étudiés à partir des galeries (API Bio Mérieux) permettant la caractérisation des espèces bactériennes au sein du même genre : si au moins cinq colonies bactériennes sont présentes, le germe isolé est retenu comme responsable de la mammite (21).

\section{Analyses statistiques}

Le test du chi 2, test non paramétrique permettant de tester l'indépendance entre variables aléatoires, a été utilisé.

\section{RESULTATS}

\section{Enquête épidémiologique}

Les résultats ont indiqué que la traite manuelle était pratiquée dans 40 p. 100 des exploitations (effectifs : 12 à 18 vaches) (tableau II) et la traite mécanique au moyen de chariots trayeurs était pratiquée dans les autres exploitations étudiées. La salle de traite était

Tableau I

Interprétation du California mastitis test

\begin{tabular}{|c|c|c|c|}
\hline Score & Aspect & Concentration cellulaire & Interprétation \\
\hline 0 & Mélange liquide sans précipitation & 0 à 200000 & Pas d'infection subclinique \\
\hline 1 & Floculat très fin qui disparaît après agitation & 150000 à 500000 & Pas d'infection subclinique \\
\hline 2 & Floculat très net sans tendance à la gélification & 400000 à 1500000 & Infection subclinique légère \\
\hline 3 & $\begin{array}{l}\text { Floculat épais avec formation d'un gel ; } \\
\text { par endroits, consistance du blanc d'œuf }\end{array}$ & 800000 à 5000000 & Infection subclinique nette \\
\hline 4 & Gel épais à la consistance du crachat & $>5000000$ & Infection subclinique parfois clinique \\
\hline
\end{tabular}

\section{Tableau II}

Caractéristiques des exploitations et résultats du California mastitis test

\begin{tabular}{lcccccc} 
Exploitation & $\begin{array}{c}\text { Mode } \\
\text { de traite }\end{array}$ & $\begin{array}{c}\text { Hygiène } \\
\text { de la traite }\end{array}$ & $\begin{array}{c}\text { Traitement } \\
\text { au tarissement }\end{array}$ & $\begin{array}{c}\text { Nb. de } \\
\text { vaches }\end{array}$ & $\begin{array}{c}\text { Nb. de vaches } \\
\text { positives (fréquence) }\end{array}$ & $\begin{array}{c}\text { Nb. de quartiers } \\
\text { positifs }\end{array}$ \\
\hline E1 & Mécanique & Lavage de la mamelle & Systématique & 48 & $16(33 \%)$ & 38 \\
E2 & Mécanique & Lavage de la mamelle & Systématique & 36 & $14(39 \%)$ & 32 \\
E3 & Mécanique & Lavage de la mamelle & Pas systématique & 29 & $10(35 \%)$ & 26 \\
E4 & Manuel & Lavage de la mamelle & Aucun & 18 & $10(35 \%)$ & 22 \\
E5 & Mécanique & Lavage de la mamelle & systématique & 39 & $8(56 \%)$ & 18 \\
E6 & Mécanique & Lavage de la mamelle & Pas systématique & 17 & $4(24 \%)$ & 14 \\
E7 & Manuel & Lavage de la mamelle & Aucun & 15 & $9(60 \%)$ & 25 \\
E8 & Manuel & Aucune & Aucun & 12 & $7(58 \%)$ & 18 \\
E9 & Mécanique & Lavage de la mamelle & Pas systématique & 20 & $9(45 \%)$ & 17 \\
E10 & Manuel & Aucune & Aucun & 14 & $9(64 \%)$ & 25 \\
Total & & & & 248 & $96(38,6 \%)$ & 235
\end{tabular}


absente dans la totalité des élevages. Une différence très significative a été notée entre la traite manuelle et la traite mécanique $(\mathrm{p}<0,001)$ quant au nombre de germes de mammites isolés. La machine à traire n'était pas désinfectée correctement et aucun contrôle de machine n'était réalisé, expliquant la plus forte prévalence des germes pathogènes dans les élevages où se pratiquait la traite mécanique.

L'analyse des informations recueillies sur l'hygiène de la traite a montré que le nettoyage de la mamelle à l'eau n'était réalisé systématiquement que dans 80 p. 100 des exploitations. Le nettoyage était le plus souvent incorrect, réalisé à mains nues à l'aide d'une éponge ou avec des serviettes en coton. L'essuyage et l'élimination des premiers jets étaient très rarement pratiqués. L'étude statistique a révélé que l'hygiène de la traite était associée aux mammites subcliniques avec une valeur significative $(\mathrm{p}<0,01)$.

Les traitements au tarissement avec un anti-infectieux étaient pratiqués dans 30 p. 100 des exploitations. Ils étaient généralement administrés par voie intramammaire. Une différence très significative a été notée entre l'application ou non d'un traitement au tarissement et l'apparition de mammites subcliniques $(\mathrm{p}<0,001)$.

\section{Test de dépistage}

La prévalence troupeau de CMT positif $(\mathrm{CMT} \geq 2)$ a varié de 24 à 64 p. 100 selon les troupeaux avec, pour l'ensemble de l'échantillon, un total de 96 vaches (38,6 p. 100) dont 235 quartiers étaient atteints. La moyenne du nombre de quartiers atteints par vache a été de 2,4 (tableau II).

\section{Analyses bactériologiques du lait}

Les examens bactériologiques ont révélé que sur les 235 échantillons de lait analysés, 186 ont été positifs (79 p. 100), 23 pluricontaminés (9 p. 100) et 26 stériles (11 p. 100). Au total, 228 isolements bactériens ont été réalisés correspondant à l'identification de sept espèces de bactéries (tableau III). Le genre Staphylococcus a dominé les isolats avec 30 p. 100 de S. aureus et 43 p. 100 de staphylocoques à coagulase négative $(\mathrm{SCN})$. Les principales espèces de SCN isolées ont été $S$. chromogenes et $S$. epidermis avec des fréquences respectives de 18,4 et 12,3 p. 100 (tableau IV).

Les germes dits de réservoir mammaire (21) ont été majoritaires et représentés par $S$. aureus, avec un taux de prévalence de 79,4 p. 100 des bactéries isolées à partir des 235 quartiers révélés positifs par le CMT. La fréquence des germes de l'environnement,

\section{Tableau III}

Espèces bactériennes isolées

$\begin{array}{lrc}\text { Bactéries isolées } & \text { Nb. } & \text { Fréquence (\%) } \\ \text { Staphylocoques à coagulase négative } & 98 & 43 \\ \text { Staphylococcus aureus } & 69 & 30,3 \\ \text { Streptococcus uberis } & 9 & 3,9 \\ \text { Streptococcus agalactiae } & 8 & 3,5 \\ \text { Streptococcus dysgalactiae } & 6 & 2,6 \\ \text { Escherichia coli } & 33 & 14,5 \\ \text { Klebsiella } & 5 & 2,2 \\ \text { Total } & 228 & 100\end{array}$

Escherichia coli, Klebsiella sp. et Streptococcus uberis, ont été respectivement de 14,5, 2,2 et 3,9, soit une fréquence globale de 20,6 p. 100.

Les cas de mammites subcliniques pour lesquelles une seule espèce a été isolée ont représenté 58,1 p. 100 des échantillons positifs. Ils ont été dominés par les SCN. Les cas d'infections multiples représentés par l'association de deux espèces ont concerné 41,9 p. 100 des échantillons positifs. Le germe le plus souvent isolé en association avec un autre a été Staphylococcus aureus.

\section{DISCUSSION}

\section{Enquête épidémiologique}

L'hygiène de la traite a été jugée globalement déficiente dans la plupart des exploitations suivies. Le simple lavage des trayons était négligé, parfois pratiqué à l'aide d'une lavette collective avec de l'eau uniquement et sans être suivi d'essuyage. L'élimination des premiers jets avant la traite se faisait généralement sur le sol sous la vache, présentant ainsi un facteur de risque de contamination de la surface de couchage de la vache. Autant de fautes d'hygiène et de carences qui constituent des facteurs de risques des mammites subcliniques $(17,20)$. Les observations de la présente étude sont en accord avec celles de plusieurs autres auteurs $(4,12)$ qui indiquent que ces facteurs sont étroitement liés à l'installation des infections mammaires.

\section{Test de dépistage}

Le dépistage des mammites subcliniques est à la base de leur contrôle. Dans la présente étude, l'utilisation du CMT a permis de détecter une fréquence de 79 p. 100 de mammites subcliniques. A titre de comparaison, une étude récente réalisée par Aggad et coll. (1) portant sur l'évaluation de la qualité hygiénique du lait dans l'Ouest algérien a montré que les mammites étaient décelées dans 76 p. 100 des laits de mélange et 47 p. 100 des laits individuels. Une étude des mammites des vaches laitières des hautes terres de Madagascar, réalisée à la ferme à l'aide du CMT et du papier pH, a montré que 43 p. 100 des vaches avaient un lait positif à ces tests (21).

Selon Hanzen (16), le CMT, lorsqu'il est réalisé régulièrement, présente les mêmes indications que le comptage cellulaire individuel. Il a l'avantage, par rapport à ce dernier, d'être moins coûteux, de pouvoir être réalisé par tous les éleveurs et de fournir une image plus précise des infections en donnant des résultats

$\begin{array}{lcc}\text { Espèce bactérienne } & \text { Nb. } & \text { Fréquence (\%) } \\ \text { Staphylococcus chromogenes } & 42 & 18,4 \\ \text { Staphylococcus epidermis } & 28 & 12,3 \\ \text { Staphylococcus xylosus } & 10 & 4,4 \\ \text { Staphylococcus warneri } & 7 & 3 \\ \text { Staphylococcus haemolyticus } & 10 & 4,4 \\ \text { Staphylococcus cohnii } & 1 & 0,4 \\ \text { Total } & 98 & 43\end{array}$


quartier par quartier. Il peut également être utilisé pour vérifier, voire sélectionner les animaux à traiter au moment du tarissement. Dans la pratique, le CMT constitue donc la méthode de choix pour la détection des mammites subcliniques par l'éleveur (15).

\section{Analyses bactériologiques du lait}

Les résultats de l'analyse bactériologique ont révélé que, sur les 235 échantillons de lait récoltés, 212 ont réellement pu être analysés au laboratoire et 186 (79 p. 100) ont été positifs. Par ailleurs, 11 p. 100 des prélèvements on été trouvés indemnes de bactéries après culture. Plusieurs raisons pouvaient l'expliquer : le germe était enkysté dans le parenchyme mammaire, cas de S. aureus (2); une antibiothérapie préalable a inhibé les germes sans les détruire complètement; les prélèvements ont été mal conservés. Pour des raisons pratiques un certain nombre d'échantillons ont été congelés. Certains germes supportent mal la congélation, comme Escherichia coli ou les SCN, tandis que d'autres la supportent bien, comme les streptocoques ou S. aureus (23).

Les SCN ont représenté 43 p. 100 des bactéries isolées. Ce sont les agents étiologiques les plus fréquemment rencontrés dans le cas de mammite subclinique. Ces germes ont longtemps été considérés comme des agents pathogènes rarement responsables de ce type d'infection (13), mais les recherches réalisées au cours des dix dernières années font apparaître l'importance des SCN comme germes pathogènes responsables de mammites cliniques et subcliniques $(4,7,24)$. Ils sont maintenant reconnus comme étant les agents responsables majeurs des infections intramammaires bovines.

Le nombre élevé de ces germes serait dû aux mauvaises conditions d'hygiène de la traite. Parmi les SCN, Staphylococcus chromogenes a occupé la première place dans la présente étude comme agent responsable de mammite subclinique, suivi par S. epidermis, (tableau IV), bien que ce dernier ait été le germe le plus souvent rencontré dans d'autres études $(4,8)$.

Classé parmi les agents pathogènes majeurs, $S$. aureus est, après les $\mathrm{SCN}$, le germe le plus fréquemment isolé des quartiers infectés, probablement en relation avec des déficiences en matière d'hygiène (9). Il a été isolé avec une fréquence de 30,3 p. 100 dans la présente étude, le plus souvent lors d'infections multiples. Il se trouve en grand nombre dans le lait cru. Son réservoir est constitué par les glandes mammaires infectées mais aussi par le portage cutané des animaux sains. Wallemacq et coll. (25) indiquent que les mammites à $S$. aureus sont considérées comme l'une des maladies majeures chez les vaches laitières, causant de lourdes pertes économiques principalement dues à la réduction de la qualité et de la quantité de lait produit. Elles se caractérisent fréquemment par des formes subcliniques et chroniques, rendant leur diagnostic et leur contrôle difficile. En 2007, Nagahata et coll. (18) signalent que $S$. aureus est détecté avec une fréquence de 41,2 p. 100 chez 54 vaches en lactation au Japon.

Escherichia coli est considéré comme un germe de l'environnement et il est présent en abondance sur tous les supports des étables et dans l'eau. Il a été isolé avec une fréquence de 14,5 p. 100 dans la présente étude ; le mauvais entretien de la litière et la mauvaise hygiène de la stabulation et des vaches en général pouvaient expliquer sa présence. Une étude effectuée à Madagascar (21) rapporte qu'E. coli est isolé dans 20 p. 100 des laits de mammites. Ce germe, responsable le plus souvent de mammites cliniques aiguës, occupe aussi une place importante dans les infections intramammaires en France avec une fréquence de 14,5 p. 100 dans une étude de 1991 (13).

\section{CONCLUSION}

Le présent travail a permis d'évaluer la prévalence des mammites subcliniques qui demeurent parmi les pathologies dominantes sévissant dans les élevages laitiers en Algérie et d'identifier les germes qui en sont responsables. Cette pathologie multifactorielle représente un trouble majeur chez la vache laitière, affectant la qualité du lait et le bien-être animal. La maîtrise des mammites subcliniques devrait impliquer un dépistage systématique et précoce de ces affections à l'aide d'un test rapide et fiable comme le CMT, des conditions de traite et d'hygiène préservant l'intégrité de la mamelle, une utilisation raisonnée des anti-infectieux, et l'identification des agents pathogènes responsables de ces infections. Compte tenu du faible nombre d'exploitations échantillonnées, une étude portant sur un plus grand nombre d'exploitations est suggérée pour confirmer les résultats présentés ainsi qu'une caractérisation plus poussée des germes isolés en recherchant leurs éventuelles antibiorésistances.

\section{BIBLIOGRAPHIE}

1. AGGAD H., MAHZOUZ F., AHMEDAMMAR Y., KIHAL M., 2009. Evaluation de la qualité hygiénique du lait dans I'ouest algérien. Rev. Méd. vét., 169 : 590-595.

2. ALEXANDRE A., 2005. Utilisation des comptages cellulaires dans la comparaison de deux préparations hors lactation. Thèse Doct. vét., Université Claude Bernard, Lyon I, France, p. 73-74.

3. AMELLAL R., 1995. La filière lait en Algérie : entre I'objectif de la sécurité alimentaire et réalité de la dépendance. Options Méditerr., Sér. $B:{ }^{\circ} 14$.

4. BEN HASSEN S., MESSADI L., BEN HASSEN A., 2003. Identification et caractérisation des espèces de Staphylococcus isolées de lait de vaches atteintes ou non de mammite. Ann. Méd. vét., $147: 41-47$.

5. BENNET G., 1993. What to expect from sire selection to lower somatic cell count. In: 32nd Annual Meeting National Mastitis Council, Kansas City, USA, 4-7 Oct. 1993, p. 65-72.

6. BEROUAL K., 2003. Caractérisation des germes d'origine bactérienne responsables des mammites bovines dans la région de la Mitidja. Thèse Magister, Université de Blida, Algérie, 134 p.

7. BES M., GUERIN-FAUBLEE V., MEUGNIER H., ETIENNE J., FRENEY J., 2000. Improvement of the identification of staphylococci isolated from bovine mammary infections using molecular methods. Vet. Microbiol., 71: 287-294.

8. BIGERSON A., JONSSON P., HOLMBERG O., 1992. Species identification and some characteristics of coagulase-negative staphylococci isolated from bovine udders. Vet. Microbiol., 31: 181-189.

9. BONNIER M., DORE C., MEDEO J., GUERIN-FAUBLEE V., 2006. Activité in vitro de la tylosine et de la tilmicosine sur des coques isolés de mammites bovines. Rev. Méd. vét., 157 : 486-489.

10. BOUTET P., DETILLEUX J., MOTKIN M., DELIEGE M., PIRAUX E., DEPINOIS A., DEBLIQUY P., MAINILJ., CZAPLICKI G., LEKEUX P., 2005. Comparaison du taux cellulaire et de la sensibilité antimicrobienne des germes responsables de mammite subclinique bovine entre les filières conventionnelle et biologique. Ann. Méd. vét., 149 : 173-182.

11. BRADLEY A.J., 2002. Bovine mastitis: An evolving disease. Vet. J., 164: 116-128.

12. DELFOSSE C., FOIDMONT E., CURNEL Y., HUMBLET M.F., HANZEN C., BEROTOZZI C., BARTIAUX-THILL N., 2006. Etude éco pathologique des facteurs de risque des mammites dans les élevages laitiers en Wallonie. Renc. Rech. Ruminants, 13: 440.

13. FABRE J.M., BERTHELOT X., LEBRET P., BLANC M.F., BLANC M.C., 1991. Estimation de la fréquence des différents germes responsables d'infection mammaires en élevage bovin laitier dans le sud-ouest de la France. Rev. Méd.vét., 142 : 823-829.

14. faroult B., POUTREL B., BROUILLET P., LE PAGE P., 2003. Mammite des bovins (cliniques et subcliniques) : démarche diagnostique et thérapeutique. Dépêche vét. (suppl.) : 87. 
15. GAMBO H., AGNEM ETCHIKE C., 2001. Dépistage de mammites subcliniques chez des vaches Goudali en lactation au Nord Cameroun. Rev. Elev. Méd. vét. Pays trop., 54 : 5-10.

16. HANZEN C., 2009. Propédeutique de la glande mammaire : sémiologie, diagnostic individuel et de troupeau. Liège, Belgique, Université de Liège, p. 11.

17. MTAALLAH B., OUBEY Z., HAMMAMI H., 2002. Estimation des pertes de production en lait et des facteurs de risques des mammites subcliniques à partir des numérations cellulaires de lait de tank en élevage bovin laitier. Rev. Méd. vét., 153 : 251-260.

18. NAGAHATA H., ITO H., MARUTA H., NISHIKAWA Y., SUSKINO H., MATSKI S., HIGUCHI H., OKUHIRA A., ANRI A., 2007. Controlling highly prevalent Staphylococcus aureus mastitis from dairy farm. J. vet. med. Sci., 69: 893-898.

19. NATIONAL MASTITIS COUNCIL, 1999. Laboratory handbook on bovine mastitis, rev. Edn. Madison, WI, USA, National Mastitis Council.

20. PLUVINAGE P., DUCRUET T., JOSSE J., MONICAT F., 1991. Facteurs de risques des mammites des vaches laitières. Résultats d'enquête. Rec. Méd. vét., 167 : 105-112.

\section{Summary}

Boufaida Asnoune Z., Butel M.J., Ouzrout R. Prevalence of major bacteria causing subclinical mastitis in dairy cows in Northeastern Algeria

Bacterial analyses of 235 milk samples of dairy cattle distributed over 10 farms in Northeastern Algeria, affected by subclinical mastitis diagnosed by the California mastitis test, were carried out over a two-year period (2008-10). Bacterial isolation concerned $80 \%$ of the samples. Among the pathogenic agents detected, Gram-positive cocci were the most frequently found, particularly coagulase-negative staphylococci (43\%) and Staphylococcus aureus (30\%). Escherichia coli was isolated in $14 \%$ of the samples. The other bacteria isolated were Streptococcus uberis (4\%), Streptococcus agalactiae (3\%), Streptococcus dysgalactiae (3\%), and more rarely Klebsiella sp. $(2 \%)$.

Keywords: Cattle - Dairy cow - Mastitis - Latent infection Bacteriological analysis - Algeria.
21. RAKOTOZANDRINDRAINY R., FOUCRAS G., 2007. Etiologie bactérienne des mammites des vaches laitières du triangle laitier des hautes terres de Madagascar. Rev. Méd. vét., 158 : 106-110.

22. REMY D., 2010. Les mammites : hygiène, prévention, environnement, $1^{\text {re }}$ édn. Paris, France, La France agricole, 260 p.

23. SCHUKKEN Y.H., GROMMERS F.J., SMIT J.A., VANEGEER D., BRAND A., 1989. Effect of freezing on bacteriologic culturing of mastitis milk samples. J. Dairy Sci., 72: 1900-1906.

24. TAPONEN S., 2008. Bovine mastitis caused by coagulase-negative staphylococci. Accad. Diss., Faculty of Veterinary Medicine, University of Helsinki, Finland, $63 \mathrm{p}$

25. WALLEMACQ H., GIRARD B., LEKEUX P., BUREAU F., 2010. La vaccination contre les mammites à Staphylococcus aureus chez la vache laitière. Ann. Méd. vét., 154 : 16-29.

Accepté le 18.06.2012

\section{Resumen}

Boufaida Asnoune Z., Butel M.J., Ouzrout R. Prevalencia de las principales bacterias causantes de la mastitis subclínica en las vacas lecheras en el noreste de Argelia

Los análisis bacteriológicos de 235 muestras de leche de vacas lecheras con mastitis subclínica detectadas por el California Mastitis Test, provenientes de diez fincas en el noreste de Argelia, se llevaron a cabo durante un período de dos años (2008-2010). El aislamiento de la bacteria afectó al 89\% de las muestras. Entre las bacterias aisladas, los cocos Gram positivos fueron los más frecuentes, especialmente los estafilococos coagulasa negativos (43\%) y los Staphylococcus aureus (30\%). El Escherichia coli se aisló en el 14\% de las muestras. Otros microorganismos aislados fueron los Streptococcus uberis (4\%), los Streptococcus agalactiae (3\%), los Streptococcus dysgalactiae (3\%), y más raramente, la Klebsiella sp. (2\%).

Palabras clave: Ganado bovino - Vaca lechera - Mastitis Infección latente - Análisis bacteriológico - Algeria. 
